\title{
Capítulo 16. Utilidad del mapeo tridimensional en la ablación en pacientes pediátricos
}

\section{Chapter 16. Usefulness of three-dimensional mapping in pediatric patients}

\author{
Álvaro Arenas \\ Fundación Cardioinfantil-Instituto de Cardiología, Bogotá, Colombia
}

Recibido el 17 de noviembre de 2015; aceptado el 18 de enero de 2016

\section{Introducción}

La electrofisiología pediátrica invasiva fue fundada sobre los principios desarrollados en la práctica de electrofisiología de adultos, pero siempre ha abarcado un espectro diferente de condiciones y desafíos; entre estos, por ejemplo, el efecto de un corazón de menor tamaño ha requerido el desarrollo de catéteres más pequeños. La alta frecuencia de anormalidades electrofisiológicas y problemas en la población infantil con reparación o paliación de cardiopatías congénitas, presenta problemas únicos, así como oportunidades para el desarrollo de herramientas y técnicas para el mejoramiento de los estudios electrofisiológicos invasivos en esta población. El avance de las tecnologías de mapeo y la ablación, ha hecho que el tratamiento de las arritmias en niños sea más seguro y eficaz ${ }^{1,2}$.

El método estándar para la inserción y la navegación del catéter es la fluoroscopia, que en niños tiene un potencial de efectos secundarios relacionados con la dosis: carcinogénesis, eritema y necrosis cutánea, cataratas y posibles efectos genéticos. La exposición de radiación requerida para que estos efectos se produzcan es relativamente alta y rara vez vista con operadores experimentados y con los sistemas con- temporáneos y el uso de pulsos de fluoroscopia. Sin embargo, los niños son organismos en crecimiento, con un período de vida más largo, que permite mayor tiempo potencial para desarrollar neoplasias y otros efectos secundarios ${ }^{3}$. De igual forma, los niños sometidos a cateterismo cardiaco tienen riesgo elevado de desarrollar lesiones inducidas por la radiación resultante de la mayor sensibilidad a ésta en sus tejidos en comparación con los de los adultos (la sensibilidad a la radiación está relacionada con la tasa de división celular en un tejido dado). Así mismo, los niños con cardiopatías congénitas complejas tienen mayor riesgo debido a la necesidad frecuente de realizar cateterismos cardíacos durante la infancia, unidos al aumento de la duración (y la carga de radiación) de procedimientos intervencionistas complejos; adicionalmente, algunos de los tejidos sensibles a las lesiones por radiación (ojos, tiroides, y gónadas) están más cerca del corazón en los niños pequeños que en los adultos, característica que los hace más próximos al generador y a los rayos dispersos $^{4}$. Los pacientes de menor tamaño y con mayor frecuencia cardiaca, también requieren mayor magnificación y altas frecuencias de cuadros, todo lo cual contribuye a un incremento significativo en la dosis de radiación. Igualmente, puede ser necesario repetir los procedimientos en cierto número 
de casos, debido a fallas primarias o recurrencia de las mismas o un sustrato arrítmico diferente. Varios registros han demostrado que el mayor número de complicaciones, así como el tiempo de procedimiento y de fluoroscopia, se producen cuando se realizan intervenciones a menores de 5 años ${ }^{5}$. Por estas razones, un sistema de navegación no fluoroscópica de los catéteres, puede proporcionar beneficios significativos a la población pediátrica ${ }^{6,7}$.

\section{Utilidad del mapeo tridimensional}

Los sistemas de mapeo tridimensional no fluoroscópicos tienen características de: navegación intracardiaca no fluoroscópica que permiten reconstruir en forma geométrica tridimensional las cavidades cardiacas, hacer seguimiento de los catéteres en tiempo real y mapear la arritmia por codificación de colores, al definir la precocidad, reconstruir la secuencia de activación de la arritmia y mostrar la amplitud del voltaje del tejido estudiado ${ }^{2}$, lo que lleva a realizar un estudio más detallado de las arritmias y obtener diferentes mapas (activación, propagación, voltaje y anatómico) al obtener mejores resultados en la ablación y disminuir de manera significativa la dosis de radiación ${ }^{8,9}$.

En cuanto al mecanismo de las arritmias, la mayoría de las taquicardias en la población pediátrica sin cardiopatía congénita son supraventriculares y de estas las más frecuentes son las taquicardias por reentrada aurículo-ventricular, facilitadas por una vía accesoria (incluyendo el síndrome de WolffParkinson-White); le siguen en frecuencia la taquicardia por reentrada nodal aurículo-ventricular y la taquicardia auricular focal. Las de reentrada aurículo-ventricular son la forma de presentación más común en los grupos de recién nacidos y lactantes, en tanto que las de reentrada nodal aurículoventricular son poco habituales en la primera infancia y aumentan a medida que lo hace la edad, semejando los patrones observados en la práctica de adultos. Se ha demostrado que el uso de sistemas de mapeo tridimensional en pacientes pediátricos con taquicardia por reentrada nodal aurículo-ventricular disminuye el riesgo de bloqueo aurículoventricular. Otros mecanismos menos comunes incluyen la taquicardia ectópica de la unión y la taquicardia permanente reciprocante de la unión, las cuales pueden ser incesantes y llevar a disfunción ventricular y/o miocardiopatía dilatada. El flutter auricular rara vez se encuentra fuera del grupo de edad neonatal, excepto en los postoperados de cardiopatías congénitas $^{9,10}$.

Los sistemas de mapeo tridimensional ayudan a identificar la localización de las vías accesorias aurículo-ventriculares al realizar mapas de activación (auricular o ventricular) que permiten determinar la dirección de estas y mejorar así los resultados de la ablación ${ }^{3,10}$. En los niños, las taquicardias auriculares generalmente son focales; mediante el sistema convencional fluoroscópico requieren el uso de múltiples catéteres para identificar el origen. Adicionalmente, con los sistemas tridimensionales es posible disminuir significativamente el número de catéteres (por tanto accesos venosos) y detallar con precisión el foco de la arritmia ${ }^{11}$. Las figuras $1 \mathrm{y}$ 2 muestran mapas tridimensionales de taquicardia auricular y vía accesoria.

La taquicardia ventricular puede observarse a cualquier edad durante la infancia, con un pico que ocurre en los lac-

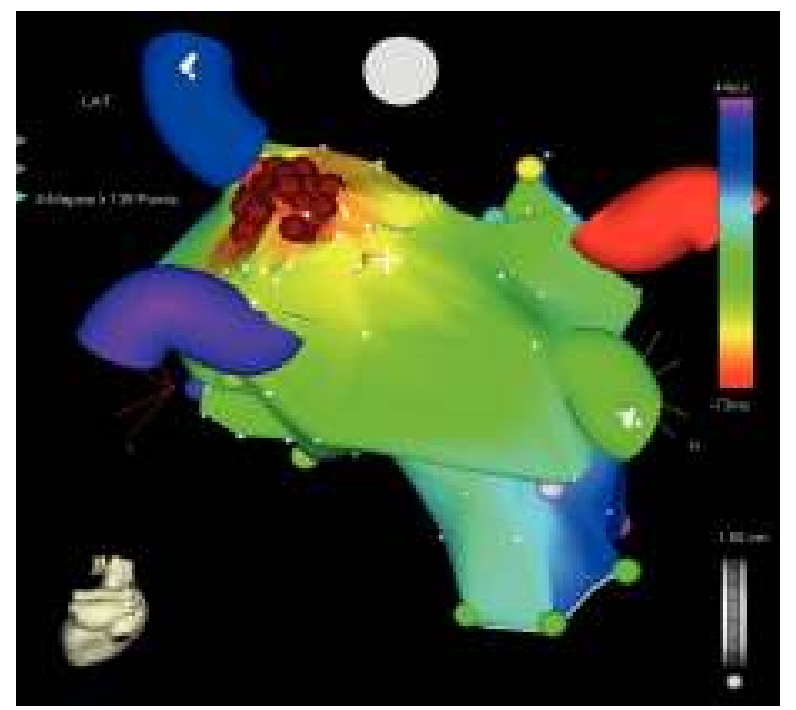

Figura 1 Reconstrucción tridimensional de la aurícula izquierda, mediante sistema CARTO, en un paciente de 6 años con taquicardia auricular focal.

tantes, y si bien la incidencia general es mucho menor que la de las arritmias supraventriculares, la evolución en general es favorable, sobre todo cuando se presenta en el primer año de vida; en muchas oportunidades no requiere tratamiento específico sino seguimiento clínico y se resuelve de manera espontánea ${ }^{12}$. La taquicardia ventricular idiopática es aquella que ocurre en corazones estructuralmente sanos; es la más frecuente dentro del grupo de las taquicardias ventriculares en la etapa escolar y adolescente, en las que predominan la del fascículo posteroinferior, seguida por la del anterosuperior y la del tracto de salida del ventrículo derecho ${ }^{13}$. Las fi-

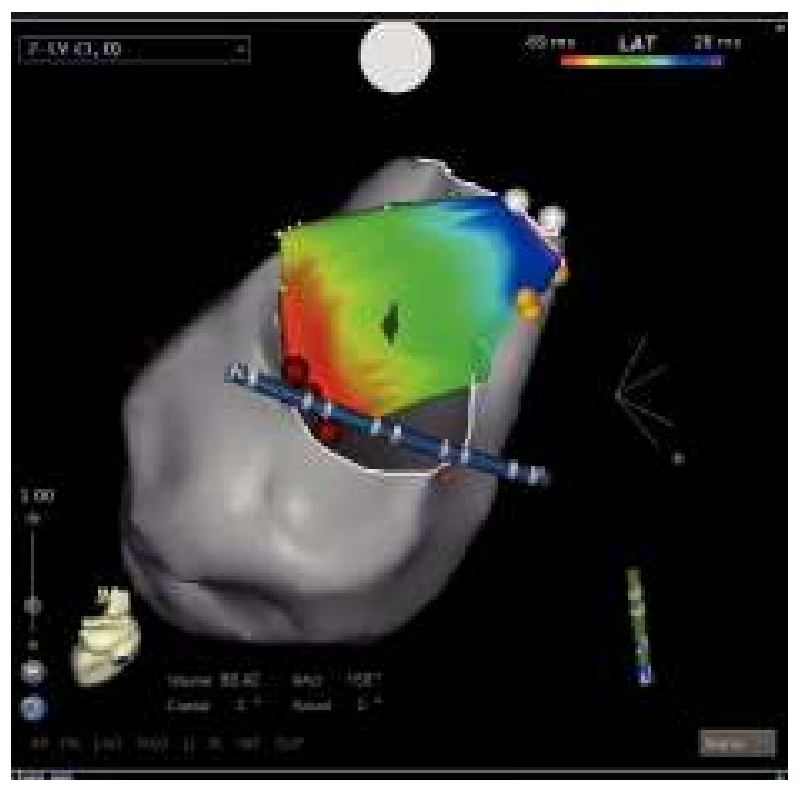

Figura 2 Reconstrucción tridimensional del ventrículo izquierdo y el anillo mitral mediante el sistema CARTO 3, con toma de puntos de activación, en un paciente con presencia de una vía accesoria posterior izquierda. 
guras 3 y 4 muestran mapas tridimensionales de pacientes con taquicardia ventricular.

En pacientes con cardiopatías congénitas las vías accesorias se asocian con anomalía de Ebstein, L-transposición de grandes vasos, miocardiopatía hipertrófica, atresia tricuspídea y doble salida del ventrículo derecho ${ }^{7}$. En los pacientes postoperados de cardiopatías congénitas los mecanismos de la arritmia varían de acuerdo con el defecto anatómico subyacente y el método de reparación quirúrgica. La fisiopatología eléctrica implica una interacción compleja entre la anatomía cardiaca anormal, el aumento de tamaño de las cavidades cardiacas por sobrecarga de presión y/o volumen, la lesión celular por la hipoxia, el bypass cardiopulmonar, la fibrosis en las líneas de sutura y parches y el trauma directo a los tejidos de conducción especializados. Las arritmias más vistas en estos pacientes son las taquicardias por reentrada intra-atrial, también conocidas como flutter incisional, que ocurren principalmente después de cirugía de Fontan en ventrículos únicos, corrección tipo Mustard y Senning en pacientes con Dtransposición de grandes vasos y en cierre de comunicación interauricular. Aunque mucho menos común que la taquicardia por reentrada intraatrial, la fibrilación auricular se puede desarrollar en un subgrupo de pacientes con cardiopatías congénitas que tienen condiciones que llevan a la dilatación marcada de la aurícula izquierda, como defectos no reparados del tabique auricular, enfermedades valvulares del lado izquierdo y disfunción del ventrículo sistémico ${ }^{12}$. En estos pacientes las definiciones anatómicas, las cicatrices subyacentes y los mecanismos de la arritmia pueden ser determinados con exactitud por los sistemas de mapeo tridimensional.

Las taquicardias ventriculares se desarrollan con mayor frecuencia en quienes les ha realizado ventriculotomía o colocado un parche para corregir un defecto interventricular (como en la reparación de la tetralogía de Fallot). El mecanismo principal de estas taquicardias es la reentrada, en la que el circuito reentrante se desarrolla cerca de la región de la cicatriz quirúrgica. Por otra parte, pueden desarrollarse en forma secundaria a un proceso miopático más generalizado sin requerir cicatrices ventriculares (p. ej., enfermedad valvular aórtica crónica o falla ventricular derecha después de la corrección tipo Mustard y Senning), en las que el estrés hemodinámico por largo tiempo causa grados avanzados de hipertrofia ventricular o disfunción global ${ }^{14}$. Uno de los puntos fundamentales para la ablación exitosa de estas taquicardias, es la identificación exacta de la anatomía y las cicatrices secundarias, en donde es invaluable la ayuda de un mapa tridimensional.

La ablación con catéter se ha aplicado con éxito a la mayoría de las formas de taquicardia en los pacientes con corazón estructuralmente normal y asociados a cardiopatías congénitas. El principal desafío durante estos casos es la distorsión de la anatomía que invalida las referencias fluoroscópicas habituales y complica la manipulación del catéter. Se deben conocer todos los detalles de la enfermedad estructural de base y de la cirugía, antes de la intervención. Los sistemas de mapeo tridimensional también se han utilizado para la ablación de los sustratos de taquicardia en los pacientes que han sido objeto de reparación quirúrgica de cardiopatías congénitas, y han mejorado de manera notoria las tasas de éxito en estos $\operatorname{casos}^{14-16}$. Se ha demostrado que con el empleo del mapeo tridimensional se aumenta la eficiencia en el tratamiento de las taquicardias auriculares así como de las ventriculares idiopáticas, un 80 a $94 \%$ y en 78 a $98 \%$ de los casos en la población pediátrica, respectivamente ${ }^{17}$.

En resumen, con el mapeo tridimensional es posible ${ }^{16}$ :

- Reconstruir la anatomía de la cavidad cardiaca en forma tridimensional.

- Visualizar los catéteres en tiempo real.

- Marcar puntos específicos.

- Definir el mecanismo de la arritmia.

- Determinar tiempos de activación y precocidad.

- Volver con precisión a puntos determinados.

- Usar menos catéteres.

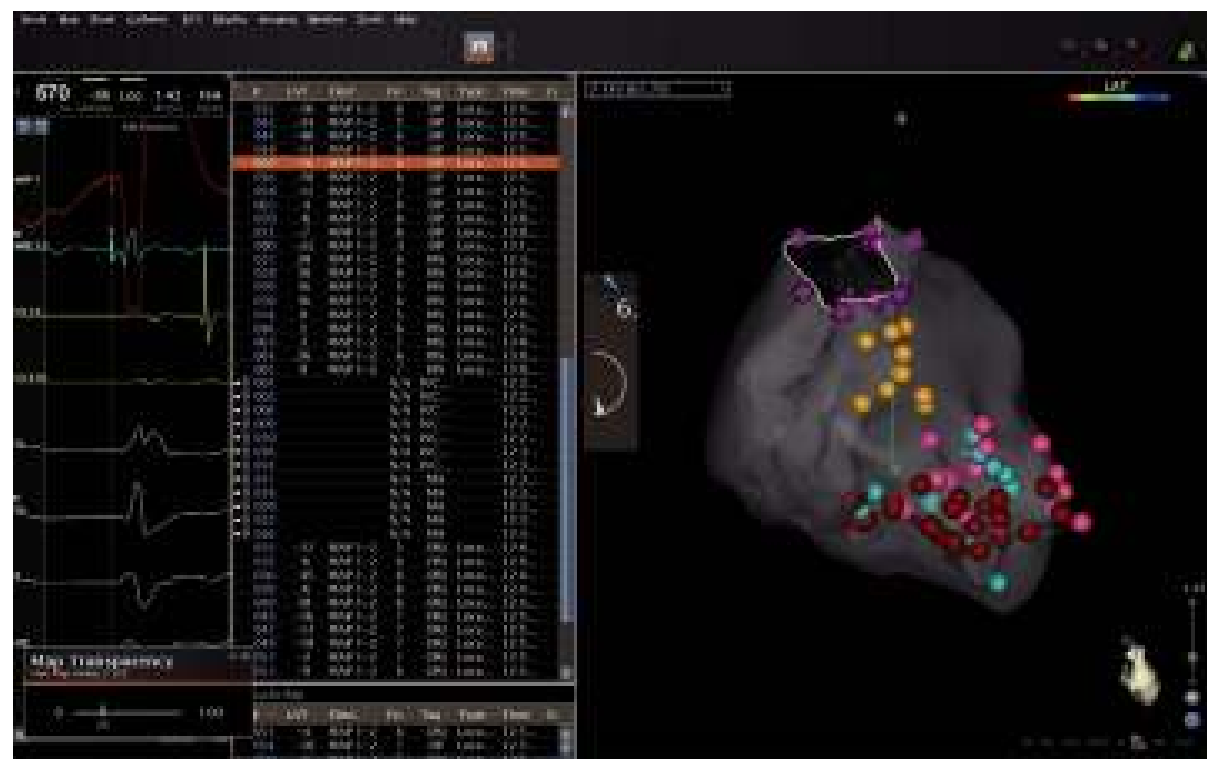

Figura 3 Mapa tridimensional del ventrículo izquierdo con sistema CARTO 3, has de His (puntos amarillos), fascículo posteroinferior (puntos azules) y potenciales diastólicos (puntos rosados) en un paciente con taquicardia ventricular fascicular posteroinferior, en quien se realizó transección del fascículo. 


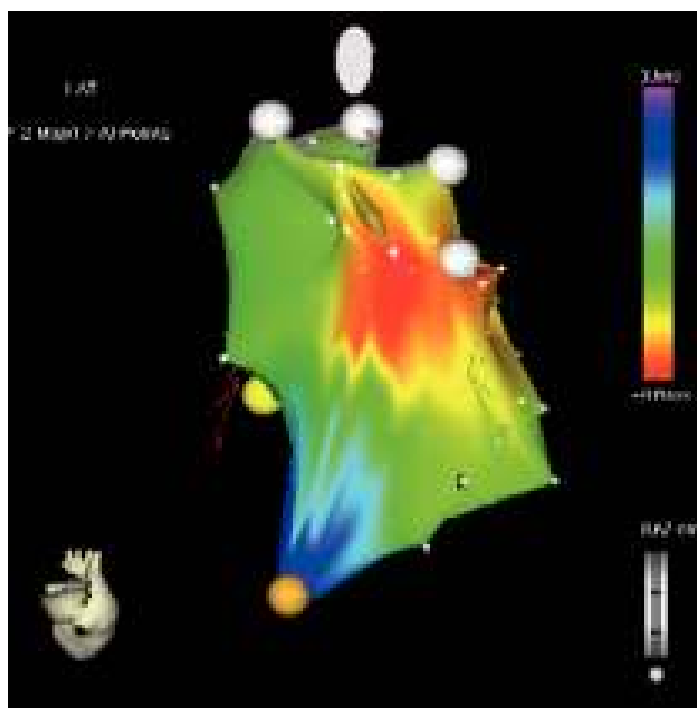

Figura 4 Reconstrucción tridimensional del tracto de salida del ventrículo derecho mediante sistema CARTO 3 en un paciente con taquicardia ventricular idiopática de este origen.

- Disminuir el tiempo de procedimiento.

- Reducir el tiempo de fluoroscopia.

- Definir cicatrices y zonas de necrosis.

- Delimitar líneas de ablación.

\section{Recomendaciones}

\section{Clase I}

- El mapeo tridimensional para ablación por radiofrecuencia está indicado cuando se desee disminuir o evitar la exposición a radiación por rayos $\mathrm{X}$ (p. ej., en pacientes menores de 4 años o de menos de $15 \mathrm{~kg}$ ) (nivel de evidencia C).

- El mapeo tridimensional para ablación por radiofrecuencia está indicado en pacientes con cardiopatías congénitas complejas (nivel de evidencia C).

- El mapeo tridimensional para ablación por radiofrecuencia está indicado en pacientes con taquicardias postoperatorias (nivel de evidencia $\mathrm{C}$ ).

- El mapeo tridimensional para ablación por radiofrecuencia está indicado en pacientes con recidivas luego de ablación convencional (nivel de evidencia C).

- El mapeo tridimensional para ablación por radiofrecuencia está indicado en pacientes con sustrato arrítmico cercano al sistema de conducción eléctrico (por ejemplo, vías anómalas parahisianas) (nivel de evidencia C).

\section{Clase IIA}

- El mapeo tridimensional para ablación por radiofrecuencia está indicado en pacientes con taquicardias auriculares (nivel de evidencia $C$ ).

- El mapeo tridimensional para ablación por radiofrecuencia está indicado en pacientes con taquicardias ventriculares (nivel de evidencia C).

- El mapeo tridimensional para ablación por radiofrecuencia está indicado en pacientes con taquicardias secundarias a vías accesorias tipo Mahaim o taquicardia permanente reciprocante de la unión (nivel de evidencia C).

\section{Bibliografía}

1. Van Hare GF, Dubin A, Collins KK. Invasive electrophysiology in children: state of the art. J Electrocardiol. 2002;35 Suppl:165-74.

2. Young ML. New techniques of mapping and ablation for tachyarrhythmias in children. Future Cardiol. 2008;4:321-31.

3. Papagiannis J, Tsoutsinos A, Kirvassilis G, et al. Nonfluoroscopic catheter navigation for radiofrequency catheter ablation of supraventricular tachycardia in children. PACE. 2006;29:971-8.

4. Justino $\mathrm{H}$. The ALARA concept in pediatric cardiac catheterization: techniques and tactics for managing radiation dose. Pediatr Radiol. 2006;36 Suppl 2:146-53.

5. Kugler J, Danford D, Houston K, Felix G; Pediatric Radiofrequency Ablation Registry of the Pediatric Radiofrequency Ablation Registry of the Pediatric Electrophysiology Society. Pediatric radiofrequency catheter ablation registry success, fluoroscopy time, and complication rate for supraventricular tachycardia: comparison of early and recent eras. J Cardiovasc Electrophysiol. 2002;13:336-41.

6. Gellis LA, Ceresnak SR, Gates GJ, Nappo L, Pass RH. Reducing patient radiation dosage during pediatric SVT ablations using an "ALARA" radiation reduction protocol in the modern fluoroscopic era. Pacing Clin Electrophysiol. 2013;36:688-94.

7. Beach C, Beerman L, Mazzocco S, Brooks MM, Arora G. Use of three-dimensional mapping in young patients decreases radiation exposure even without a goal of zero fluoroscopy. Cardiol Young. 2015. Disponible en: http://dx.doi.org/10.1017/ S1047951115002449.

8. Dieks JK, Müller MJ, Schneider HE, Krause U, Steinmetz M, Paul T, et al. Catheter ablation of pediatric focal atrial tachycardia: ten-year experience using modern mapping systems. Pediatr Cardiol. 2015 [Epub ahead of print].

9. Abrams DJ. Invasive electrophysiology in paediatric and congenital heart disease. Heart. 2007;93:383-91.

10. Szegedi N, Zima E, Clemens M, Szekely A, Kiss RG, Szeplaki G, et al. Radiofrequency ablation of focal atrial tachycardia: Benefit of electroanatomical mapping over conventional mapping. Acta Physiol Hung. 2015;102:252-62.

11. Ceresnak SR, Nappo L, Janson CM, Pass RH. Tricking CARTO: Cryoablation of supraventricular tachycardia in children with minimal radiation exposure using the CARTO 3 system. Pacing Clin Electrophysiol. 2016;39:36-41.

12. Van Hare G. Supraventricular tachycardia. En: Gillette P. Clinical Pediatric Arrhythmias 2. Philadelphia: WB Saunders Company; 2012. p. 97-120.

13. Ozyilmaz I, Ergul Y, Akdeniz C, Ozturk E, Tanidir IC, Tuzcu V. Catheter ablation of idiopathic ventricular tachycardia in children using the EnSite NavX system with/without fluoroscopy. Cardiol Young. 2014;24:886-92.

14. Walsh EP. Interventional electrophysiology in patients with congenital heart disease. Circulation. 2007;115:3224-34.

15. Abrams DJ, Earley MJ, Sporton SC, et al. Comparison of noncontact and electroanatomic mapping to identify scar and arrhythmia late after the fontan procedure. Circulation. 2007;115:1738-46.

16. Daniel GM, Van Hare G. Catheter ablation in children and adolescents. Heart Rhythm. 2006;3:95-101.

17. Von Bergen NH, Bansal S, Gingerich J, Law IH. Nonfluoroscopic and radiation-limited ablation of ventricular arrhythmias in children and young adults: A case series. Pediatr Cardiol. 2011;32:743-7. 